\title{
¿CONSIDERAN LOS MAGALLÁNICOS QUE SU CULTURA POSEE UNA ESENCIA ÚNICA?: ESTUDIO DESCRIPTIVO DE LA APLICACIÓN DE UN INSTRUMENTO DE EVALUACIÓN DE ESENCIALISMO CULTURAL A UNA MUESTRA PUNTARENENSE
}

\author{
CLAUDIA ESTRADA GOIC" Y SERGIO AVENDAÑO ALARCÓN"*
}

\begin{abstract}
RESUMEN
La presente investigación tuvo como objetivo realizar la descripción de la presencia de creencias asociadas al esencialismo psicológico en la percepción de las características de la cultura magallánica. La muestra estuvo constituida por doscientos habitantes de la ciudad de Punta Arenas quienes se prestaron voluntariamente para participar en el estudio. Se utilizó un diseño no experimental transversal descriptivo. Para medir esencialismo se aplicó un cuestionario breve de esencialismo cultural. Los resultados indican que un alto porcentaje de los participantes poseen creencias esencialistas sobre la identidad magallánica. Algunas ideas que ilustran este resultado son la alta adhesión a afirmaciones respecto a que ser magallánico es hereditario, es parte de lo más profundo de la identidad y que debe ser protegida de otras culturas. Alguna de sus implicaciones para las relaciones inter-grupo y las perspectivas de investigaciones futuras son discutidas.
\end{abstract}

PALABRAS CLAVES: Esencialismo, cultura, identidad.

DO THE MAGELLANIC THAT THIER CULTURE HAS UNIQUE ESSENCE?: DESCRIPTIVE STUDY OF THE APLICATION OF AN INSTRUMENT OF CULTURAL ESSENTIALISM EVALUATION OF A PUNTARENESE SAMPLE

\begin{abstract}
This research aimed to make a description of the presence of beliefs associated with psychological essentialism in the characteristics' perception of the Magellan's culture. The sample consisted of two hundred habitants of the city of Punta Arenas who were provided voluntarily to participate in the study. We used a non-experimental descriptive design. A brief questionnaire was used to measure cultural essentialism. The results indicate that a high percentage of participants have essentialist beliefs about the Magellan's identity. Some ideas that illustrate this result are the high adherence to assertions regarding Magellan's
\end{abstract}

Escuela de Psicología. Universidad de Magallanes. Av. Bulnes 01855. 6200000 - Punta Arenas - Chile. E-mail: claudia. estrada@umag.cl

** Facultad de Humanidades, Ciencias Sociales y Ciencias de la Salud. Escuela de Psicología. Universidad de Magallanes. Punta Arenas - Chile 
identity is hereditary, is part of the depths part of identity and must be protected from other cultures. Some of its implications for inter-group relations and prospects for future research are discussed.

KEY WORDS: Essentialism, culture, identity.

\section{INTRODUCCIÓN}

Un niño de origen magallánico es adoptado por una familia canadiense a la edad de 6 meses. Catorce años después es posible que, aún consciente de haber sido adoptado, no conozca nada de su pasado, de la historia o las vivencias de la cultura magallánica de sus padres biológicos ni de la lengua de sus ancestros. ¿Cómo se verá así mismo, como magallánico o como canadiense?, y ¿cómo será percibido por su familia canadiense?, ¿así cómo su familia biológica magallánica? La respuesta de cada uno depende de las creencias que poseen respecto a cómo es que se construye la identidad social y cultural de una persona. Es posible que la visión de algunos sea que las personas son lo que su "esencia interior" les permite ser, mientras que otros sostendrán que las personas son el reflejo de sus experiencias, oportunidades y de las elecciones que han hecho en su vida.

Los seres humanos hemos desarrollado destrezas que nos permiten comprender y adaptarnos a nuestro ambiente social. Este proceso exige aprender los códigos que guían nuestro comportamiento social y también el desarrollo de estrategias para compensar nuestros límites cognitivos. Una de esas estrategias es la construcción de "teorías implícitas", que reagrupan parte de lo que sabemos del funcionamiento de la "realidad" y que usamos para orientarnos en este exigente y confuso mundo social. Una teoría implícita puede ser definida como una herramienta de interpretación de la realidad que se basa en un conjunto coherente de ideas respecto a cómo se organiza la personalidad, la naturaleza de las personas y/o la naturaleza de los grupos. Este estudio se interesa en una teoría implícita respecto a la naturaleza de los grupos denominada "esencialismo psicológico".

En la investigación en psicología social sobre la percepción de los grupos, investigadores han postulado que las personas tienden a recurrir a teorías para explicar cómo y por qué se forman los diferentes grupos sociales. (Hirschfeld, 1995a, 1995b; Rothbart \& Taylor, 1992; Yzerbyt, Rocher \& Schadron, 1997; Yzerbyt, Corneille \& Estrada, 2001; Haslam, Rothschild \& Ernest, 2002, etc.). Una de esas teorías es el esencialismo psicológico que indica que ciertas personas tienden a creer que existen grupos que poseen una naturaleza biológica o esencial. Más específicamente, el esencialismo puede ser definido como: "la tendencia de las personas a asignar a los objetos o a pensar que los objetos tienen una esencia o una naturaleza subyacente que hace que sean lo que son" (Medin, 1989, p.1476). En otros términos, el esencialismo sería una teoría implícita que reuniría una serie de creencias que explicarían que los grupos se forman y existen porque sus miembros poseen una "esencia" en común. Aunque existen dos corrientes diferentes que se han interesado en las causas del esencialismo, una que postula que es innato en los seres humanos (Atran, 1990; Atran, 1998; Gelman, 2005) y otra que plantea que es producto de procesos tales como la categorización social y en encuentro intergrupal (Haslam, Rothschild \& Ernst, 2002, Yzerbyt, Rocher y Schadron, 1997, Yzerbyt \& Schadron, 1996), los estudios en psicología social se han centrado principalmente en sus consecuencias.

Ya Sherif (1948) había observado que ciertas personas describen los eventos sociales en términos de la existencia de una "esencia" que es entendida como una naturaleza humana determinada, compuesta de cualidades que son inherentes a un grupo. El término esencialismo fue utilizado por primera vez por Allport (1954) refiriéndose a las causas del prejuicio. Este autor pensaba que este tipo de creencias era el resultado de tratar de explicar, usando el "principio del menor esfuerzo", las similitudes comunes en los miembros de un grupo. Posteriormente, Medin (1989) utilizó este término en su intento de explicar que permitía a la gente realizar agrupaciones de objetos o personas en una misma categoría. Sus postulados fueron más lejos de la lógica que indica que son las propiedades o características (de objetos y personas) las que definen la similitud "objetiva". Medin (1989; Murphy \& Medin, 1985) 
propuso que el concepto de similitud se basa en las concepciones ingenuas que los individuos tienen sobre la naturaleza de aquello que une a los objetos los unos con los otros. Estas concepciones no son ni "objetivas" ni fáciles de definir. En tal sentido la similitud perceptiva se explicaría por la creencia en la existencia de características profundas. Desde esta perspectiva, el esencialismo es definido como "la tendencia de las personas a asignar a los objetos o a pensar que los objetos tienen una esencia o naturaleza subyacente que hace que sean lo que son" (Medin, 1989, p.1476). Las consecuencias de la categorización esencialista es que las similitudes de superficie permiten inferir la existencia de propiedades profundas, inferencias que limitan la composición de nuestras representaciones mentales sobre una categoría social y sus miembros.

Rothbart y Taylor (1992) llevaron la noción de esencialismo al territorio de la psicología social. Su interés principal se centró en comprender el poder que las etiquetas tienen sobre el proceso de categorización. Estos autores distinguieron entre dos grandes tipos de categorías sociales: las "naturales" y las "artificiales". Mientras que las naturales son asimilables a las categorías observables en el mundo animal, las artificiales se refieren a objetos construidos con un fin determinado. Ocurre que aunque las categorías sociales son mucho más cercanas a las artificiales las personas tendemos a percibirlas como naturales, considerando que pertenecer a una determinada categoría social es el reflejo de una identidad única y objetiva. Como consecuencia, las categorías sociales son dotadas de poder inductivo (tendencia a inferir cualidades esenciales profundas sobre la base de la apariencia) y de inalterabilidad (si se pertenece a una categoría no es posible pertenecer a otra).

En base a lo propuesto por Rothbart y Taylor (1992), Yzerbyt y Schadron (1996) realizaron una descripción del denominado "síndrome esencialista", que reúne las características centrales que permiten discriminar cuándo nos encontramos frente a una categoría social "esencializada": (1) El grupo es visto como una entidad con destino común; (2) La pertenencia al grupo es exclusiva (3) Conocer el grupo o categoría social de una persona permite creer que se sabe mucho sobre ella, (4) Todas las otras características o atributos se organizan sobre esta pertenencia como foco de interpretación y coherencia. (ver también Hamilton, D., 2007; Hampton, 2007; Prentice, D. \& Millar, 2007; William \& Eberhardt, 2008)

Estrada (2003) definió "esencia" como el conjunto de características y propiedades biológicas, culturales o sociales que se encuentran a la base de un grupo social y que, siendo estable, es compartida por todos sus miembros. Sería la condición necesaria y suficiente para pertenecer a una categoría social o grupo, poseyendo las siguientes características: (1) Pre-existe al grupo y es su existencia en cada individuo lo que determina la formación del mismo. (2) Es invisible pero puede ser inferida a través de su manifestación en las similitudes observables (3) No cambia ya que es la parte mas estable del grupo. (4) Es exclusiva; cada grupo tiene la suya. (5) La esencia se transmite, (6) Su contenido es diverso, y puede ser de orden biológico, cultural o social.

Investigaciones desarrolladas por Leyens y su equipo (Leyens et al., 2000; Leyens et al., 2001; Vaes, Paladino, \& Leyens, 2002) muestran que las personas que tienen una visión esencialista tienden a percibir a los miembros de otros grupos como menos humanos que a los del propio grupo. Esto sería el resultado de creer que cada grupo tiene su esencia exclusiva por lo que si la del propio grupo es "humana" la de los otros grupos será, necesariamente "infra-humana". Este proceso podría conducir a que percibamos a una persona o grupo como ilegítimo o peligroso a causa de su "falta de humanidad" (BarTal, 1990, ver también Goff, Eberhardt, Williams \& Jackson, 2008).

El esencialismo ha sido estudiado también con relación a los estereotipos y prejuicios. Ya que este fenómeno explica la pertenencia a un grupo a partir de características fundamentales, otorga estabilidad a la existencia de los grupos y a su permanencia en el tiempo. Por esta razón, aquellos estereotipos que se basan en categorías que tienden a ser esencializadas, como aquellos basados en diferencias físicas, tienden a rigidizarse. Así, etiquetas sociales arbitrarias adquieren un sentido profundo $e$ indiscutible, pudiendo servir a justificar y racionalizar la división social existente. (Yzerbyt, Estrada, Corneille, Seron, \& Demoulin, 2003; Yzerbyt \& Rogier, 2001; Manchi, Chen, Roisman \& Hong, 2007).

$\mathrm{Al}$ momento de juzgar las diferencias entre los grupos, el esencialismo participa acentuando las diferencias (percibiéndolas como más importantes) 
y asignándola a factores más estables que aquellos que no tienen creencias esencialistas. Además, las personas esencialistas muestran menor tolerancia a la ambigüedad (Estrada, Seron \& Yzerbyt, 2003).

Las similitudes también son percibidas de manera diferente por los esencialistas ya que estos últimos tienden a proyectar más sus características $y$ actitudes a aquellos que son parte de su propio grupo. En otros términos, las personas que poseen una visión esencialista ven a los miembros de su grupo como más similares a sí mismo que las personas que no tienen este tipo de creencia. Las creencias esencialistas definen las fronteras del grupo y presume similitudes entre los miembros del mismo por lo que facilita la ocurrencia de la proyección sobre el propio grupo. (Estrada, 2003)

Conocemos poco sobre los efectos positivos de las creencias esencialistas aunque suponemos que afecta la estabilidad de lo que ha sido denominado la identidad social. La Teoría de la Identidad Social (Tajfel, Billig, Bundy, \& Flament, 1971), define la Identidad Social como la consecuencia de pertenecer a uno o más grupos sociales. Sería una parte del concepto de sí mismo que se estructura en la conexión existente entre el individuo y el grupo (Tajfel, 1981). Para que surja, el ambiente social debe haber sido organizado en grupos, mediante el proceso denominado "categorización social". Este proceso psicológico tendría dos funciones: (1) Sistematizar el ambiente para potenciar la adaptación del individuo y los grupos; (2) Definir el lugar que un individuo tiene en un ambiente social determinado. El esencialismo, en tanto que creencia sobre el origen de los grupos, daría estabilidad a la identidad social ya que ésta se establecería a partir de elementos invariables contenidos en la "esencia". Bar-Tal (1998) propone que las creencias de un grupo deberían ser entendidas como parte constitutiva de su identidad social. Cuando un grupo cree en su existencia entonces su identidad ha sido fundada y será mantenida por esta creencia. El que cada miembro del grupo se perciba como parte de él, le permite al grupo construir sus fronteras con otros grupos. Esta creencia inicial "somos un grupo" permite reconocer las similitudes entre los miembros de un mismo grupo y las diferencias con los miembros de otros grupos. Sin embargo, no todas las creencias se vuelven propiedad de un grupo sino sólo aquellas que sirven a su formación y a la mantención de su identidad. Las creencias pueden reunir normas, valores, objetivos e ideologías (sociales, políticas, religiosas, etc.) de un grupo. Cuando una creencia central está constituida por la idea de que "somos un grupo porque compartimos una esencia común", la identidad social de sus miembros es esencialista, y también su mirada y comprensión de la realidad social.

La pregunta guía de nuestra investigación refiere a si los magallánicos tienen una concepción esencialista de su identidad social. Los factores históricos indican que la identidad magallánica no habría sido fácil de establecer: puesto que la mayoría de quienes se consideran a sí mismos como magallánicos provienen de procesos de inmigración tanto nacional como extrajera, en consecuencia estos individuos adscriben a una doble pertenencia identitaria (y algunas veces hasta una doble nacionalidad). Además, la zona geográfica habitada por los magallánicos presenta una tasa de población temporal importante, constituida por aquellos que por razones laborales viven unos años en esta región. Por otra parte, las condiciones geográficas de aislamiento relativo y las climáticas, únicas en Chile, pueden haber favorecido una identificación mutua. Claro, esto último no explica por qué personas que han vivido largo tiempo en la región no se ven a sí mismas ni son vistas como parte de esta categoría social.

La identidad magallánica, aunque en sí misma no definible en términos precisos, constituiría una categoría fuerte que se acompaña de elementos estereotípicos, evidenciables en rasgos comunes, como "gustar de la carne con papas", poseer una bandera propia y aspirar a ser una "república independiente". Este estudio es una primera aproximación a comprender si esta identidad encuentra o no su fuerza en creencias esencialistas.

\section{OBJETIVO.}

La investigación tuvo como objetivo central describir la existencia de creencias esencialistas sobre la cultura magallánica.

\section{HIPÓTESIS.}

HI: Al menos un 30\% de los participantes mostrará una alta adhesión a creencias esencialistas sobre la cultura magallánica. 
HI: Al menos un 30\% de los participantes NO mostrará una alta adhesión a creencias esencialistas sobre la cultura magallánica.

El 30\% se utilizó como criterio ya que investigaciones realizadas sobre teorías implícitas similares señalan que tienden a mostrar una distribución normal en la población (Dweck, Chiu \& Hong, 1995, Estrada 2003).

\section{MÉTODO \\ PARTICIPANTES.}

La muestra no probabilística de tipo intencional estuvo conformada por 200 personas de la comuna de Punta Arenas quienes participaron voluntariamente en este estudio.

\section{INSTRUMENTO:}

Escala Cultural de Esencialismo Psicológico: Basado en las proposiciones teóricas de Rothbart y Taylor (1992) que Yzerbyt y Schadron (1996) sistematizaron en la descripción del "síndrome esencialista", se utilizó una adaptación de un cuestionario de esencialismo utilizado anteriormente para determinar el nivel de adhesión a creencias esencialistas sobre la cultura magallánica (ver Estrada, Yzerbyt \& Seron, 2004). Está compuesto de 5 enunciados con escalas tipo Likert de 6 puntos (desde $1=$ totalmente en desacuerdo a $6=$ totalmente de acuerdo). La ausencia de punto neutro es común en investigaciones sobre teorías implícitas (ver Dweck, Chiu, \& Hong, 1995). Los enunciados pretenden determinar si la categoría "cultura magallánica" se percibe como dotada de características exclusivas, como una entidad estable de pertenencia inalterable, dotada de una esencia común a todos sus miembros y de carácter inductivo. La validez de contenido de la escala original fue evaluada por el método de jueces; la validez de constructo ha sido evaluada en investigaciones anteriores mediante análisis de componente principal que indican una alta saturación de un solo factor (El constructo "esencialismo" postula la existencia de un solo factor constituido por diferentes características que convergen en el mismo fenómeno). La consistencia interna del cuestionario fue alta (alfa de Cronbach $=0.85$ ).

\section{DISEÑO:}

Se utilizó un diseño no experimental transversal descriptivo

\section{PROCEDIMIENTO}

Los participantes fueron contactados, en sus trabajos o lugar de estudio, uno a uno por los encuestadores, y se les invitó a participar en un estudio sobre la percepción de la cultura magallánica. Cada participante que aceptó participar contestó individualmente el cuestionario en una sola sesión. Finalmente se les agradeció por su participación y se les informó respecto al objetivo de la misma. Los datos fueron recolectados en un periodo temporal igual a 20 días.

\section{RESULTADOS}

El $100 \%$ de los participantes se declaró magallánico de los cuáles un 54,5\% fue de género femenino y un $45,5 \%$ de género masculino. La edad promedio de los participantes fue de 24,9 años, con un tercio de la muestra con edades menores o iguales a 20 años, otro tercio entre los 21 y los 23 años y el tercio final entre los 24 y los 60 años.

Los análisis de las respuestas pregunta por pregunta indican que frente a la afirmación:

1. "La verdadera esencia de la cultura magallánica están en aquellas personas que descienden de magallánicos y, que han nacido y crecido en Magallanes": El promedio de respuestas es igual a 3.9 (D.E.: 1,66), lo que revelaría acuerdo con esta afirmación ya que es significativamente diferente del punto neutro $\left(\neq 3.5, \mathrm{t}_{(199)}=2.99, \mathrm{p}<0,003\right)$. El 43,5\% se manifestó de acuerdo con esta afirmación, el 30\% ni de acuerdo ni en desacuerdo con la misma mientras que el $26,5 \%$ restante se muestra en desacuerdo.

2. "La cultura magallánica está tan fuertemente enraizada en nosotros que forma parte de lo más profundo de lo que cada magallánico es", el promedio de acuerdo fue de 4,4 (D.E.: 1,31), indicando un alto grado de adhesión $\left(\neq 3.5, \mathrm{t}_{(199)}=\right.$ 9,64, p< 0,0001). El 52\% de los participantes se manifestó de acuerdo con la afirmación, el 38\% ni 
de acuerdo ni en desacuerdo mientras que el 9,5\% restante declaró estar en desacuerdo.

3. "El pasado común de aquellos que comparten la cultura magallánica dificulta la integración de aquellos cuyas familias pertenecen a otras culturas", el promedio de acuerdo fue igual a 3,23 (D.E.: $1,45)$, revelando un bajo nivel de acuerdo $(\neq 3.5$, $\left.\mathrm{t}_{(199)}=-2,67, \mathrm{p}<0,008\right)$.El $44 \%$ no se manifestó ni de acuerdo ni en desacuerdo, mientras que el 34\% dijo estar en desacuerdo y un 21,5\% de acuerdo con la afirmación.

4. "La cultura magallánica se transmite de generación en generación de manera que se instala en la forma de ser de las personas", el promedio de acuerdo fue igual a 4,5 (D.E.: 1,40), indicando un alto nivel de acuerdo con la afirmación. $\left(\neq 3.5, \mathrm{t}_{(199)}\right.$ $=9,88, p<0,0001)$. Un $61 \%$ de los participantes indicó estar de acuerdo mientras que un 25,5\% ni de acuerdo ni en desacuerdo y un $13,5 \%$ en desacuerdo.

5. "La cultura magallánica puede abrirse a otras culturas y enriquecerse de las diferencias, pero siempre y cuando proteja aquellos aspectos esenciales de lo que es": El promedio de acuerdo alcanzó a un 4,8 (D.E.: 1,28), indicando un alto nivel de acuerdo $\left(\neq 3.5, \mathrm{t}_{(199)}=14,05, \mathrm{p}<0,0001\right)$. Un 68\% de los participantes indicó estar de acuerdo con esta afirmación mientras que un $24,5 \%$ no se manifestó ni de acuerdo ni en desacuerdo y el 7,5\% restante dijo estar en desacuerdo. (ver figura 1).

Los análisis sobre el puntaje total indican que la escala alcanza un nivel de confiabilidad aceptable (alfa de Cronbach $=0,85$ ). El análisis factorial indica la presencia de un factor que explica el $44 \%$ de la varianza.

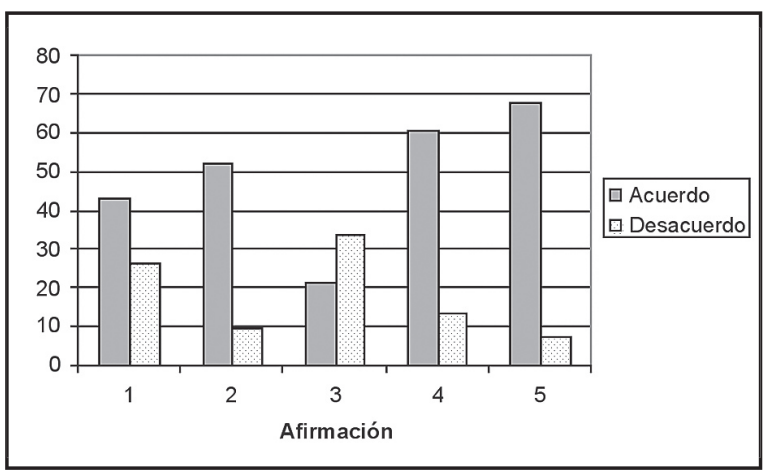

Fig. 1. Porcentaje de participantes de acuerdo y en desacuerdo con cada una de las afirmaciones.
El puntaje total muestra un promedio de esencialismo igual a 4,2 significativamente sobre el punto neutro $\left(\neq 3.5, \mathrm{t}_{(199)}=10,71, \mathrm{p}<0,0001\right)$, indicando un alto nivel de acuerdo con la visión esencialista de la cultura magallánica. La mediana es igual a 4,2 indicando que el 50\% de los participantes presenta puntajes superiores a esa cifra. El 73\% de los participantes presenta puntajes superiores al punto neutro indicando altos niveles de adhesión a una visión esencialista de la cultura magallánica (ver figura 2).

\section{DISCUSIÓN Y CONCLUSIONES}

Sobre la base de la observación de la forma en cómo los magallánicos portan su identidad (y también quienes dicen no poseer esta identidad) nos propusimos abordar la interrogante de si los así llamados magallánicos creen o no que la cultura (categoría social) posee una esencia única. En este sentido, el propósito de nuestra investigación se centró en determinar si existe una percepción esencialista de la identidad magallánica. La respuesta inicial es que los datos recogidos apoyan la hipótesis de que efectivamente los magallánicos, en su mayoría, tienen una visión esencialista, es decir, consideran la pertenencia a esta categoría social magallánicos como si se tratara del resultado de un proceso similar al de las categorías naturales.

Resulta interesante que más del 50\% de los participantes se mostró de acuerdo con la idea de que la cultura magallánica se encuentra enraizada en lo más profundo de su identidad, que dicha cultura va instalándose generación tras generación en la forma de ser de las personas y que su esencia ha de ser protegida. Estas tres creencias revelan

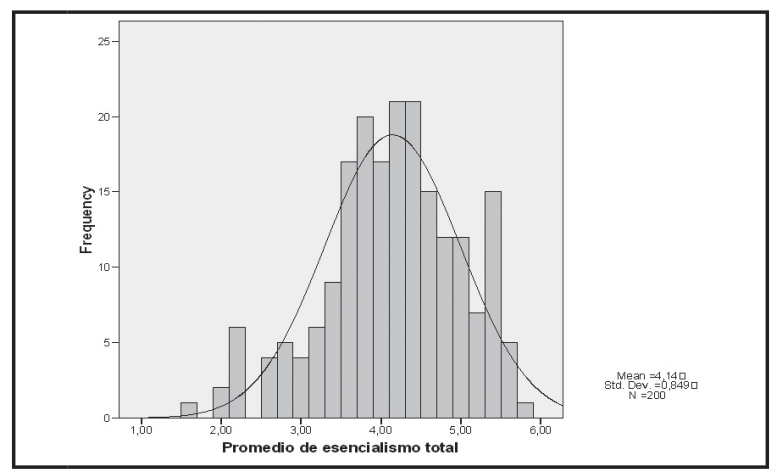

Fig. 2. 
que la concepción de ser magallánico conlleva una complejidad mayor al sólo hecho de habitar un lugar geográfico determinado. Así para estas personas, el ser magallánico supondría una esencia particular que es transmitida generación a generación como si estuviese inscrita en sus genes. Y aunque esta esencia única puede nutrirse del intercambio con otras culturas debe ser protegida dado que podría verse amenazada por ellas.

Por otra parte, el 43,5\% estuvo de acuerdo con la idea de que ser auténticamente magallánico supone ser hijo de magallánicos y haber nacido en Magallanes. Idea que -aunque en un porcentaje menor- viene a reafirmar la concepción "exclusiva" del ser magallánico; esto es, se nace como tal, por lo que no sería una esencia que pueda ser "adquirida" en el contacto con la cultura.

Aunque un porcentaje significativo (34\%) estuvo en desacuerdo con la idea (reactivo) de que las características de la cultura magallánica dificultan la integración de personas de otra cultura, no es menos cierto que uno de cada cinco participantes declara estar de acuerdo con tal idea. Este reactivo, al mismo tiempo que persigue medir el esencialismo, nos informa acerca de cómo la percepción de las diferencias entre los grupos es asociada a dificultades con relación al contacto intergrupal.

Los resultados generales van en la dirección de nuestra hipótesis inicial que proponía que al menos un 30\% de los participantes presentarían una visión esencialista de la cultura magallánica. Los análisis indican que más del $70 \%$ de los participantes muestra puntajes superiores al punto neutro y que el $50 \%$ del total tiene puntajes superiores a 4,2, siendo el puntaje máximo posible de 6,0. La identidad magallánica parece estar fundada en creencia de naturaleza esencialista que, por sus características, le otorga consistencia.

De lo hasta aquí expuesto se desprende la necesidad de comprender por qué para el magallánico ha sido necesario desarrollar estas creencias esencialistas en la construcción de su identidad. ¿Ha sido su origen multicultural el que ha impulsado el surgimiento de esta creencia como estrategia unificadora de la categoría social?, ¿Ha sido el aislamiento y su contribución a la percepción de un destino común el que ha propiciado este tipo de creencia?, etc.
El paso siguiente de nuestra investigación se orientará a la exploración de estas interrogantes así como indagará los efectos de estas creencias en diversos fenómenos de relación intergrupal tales como, la percepción de diferencias "irreconciliables" entre los magallánicos y otros grupos culturales, la convivencia cotidiana, y el surgimiento y la mantención de estereotipos resistentes con el consecuente aumento del prejuicio.

\section{BIBLIOGRAFÍA}

ALLPORT, G. 1954. The Nature of Prejudice. Reading, MA: Addison Wesley.

ATRAN, S. 1990. Cognitive foundations of natural history. New York: Cambrige University Press.

ATRAN, S. 1998. Folk biology and the anthropology of science: Cognitive universals and cultural particulars. Behavioral an Brain Sciences, 21, 547 - 609.

BAR-TAL, D. 1998. Causes and consequences of delegitimization: Models of conflict and ethnocentrism. Journal of Social Issues, 46, 65-81.

DWECK, C. S., C. CHIU Y., \& Y. Y. HONG 1995. Implicit theories. Elaboration and extension of the model. Psychological Inquiry, 6, 322-333.

ESTRADA C. 2003. L'impact de l'essentialisme psychologique sur les processus intra- et intergroupes. Tesis de doctorado MS. Université Catholique de Louvain.

ESTRADA, C., V. YZERBYT y E. SERON 2004. El efecto del esencialismo psicológico sobre las teorías ingenuas de las diferencias grupales. Psicothema, 16(2), 181-186.

GELMAN, S. 2005. The Essential Child. Oxford London Press.

GOFF, P., J. EBERHARDT, M. WILLIAMS \& M. JACKSON 2008. Not Yet Human: Implicit Knowledge, Historical Dehumanization, and Contemporary Consequences. Journal of Personality and Social Psychology. Vol. 94, No. 2, 292-306.

HAMILTON, D. 2007. Understanding the complexities of group perception: Broadening the domain. European Journal of Social psychology,37, 1077 - 1101.

HAMPTON, J. 2007. Metamorphosis: Essence, appearance, and behavior in the categorization of natural kinds. Memory \& Cognition, 35 (7), 1785-1800.

HASLAM, N., L. ROTHSCHILD y D.ERNST 2002. Are essentialist beliefs associated with prejudice? British Journal of Social Psychology, 41, 87-100.

HIRSCHFELD, L. A. 1995a. Do Children Have A Theory of Race? Cognition, 54, 209-252. 
HIRSCHFELD, L. A. 1995b. The Inheritability of Identity - Childrens Understanding.

MEDIN, D. L. 1989. Concepts and conceptual structure. American Psychologist, 44, 1469-1481.

LEYENS, J.-P. 2003. Sommes-nous tous des psychologues? Approche psychosociale des théories implicites de personnalité. Bruxelles: Mardaga.

LEYENS, J. P., V. YZERBYT y O. CORNEILLE 1996. The role of applicability in the emergence of the over attribution bias. Journal of Personality and Social Psychology, 70, 219-229.

LEYENS, J. P., A. RODRIGUEZ-PEREZ, R. RODRIGUEZTORRES, R. GAUNT, M. P. PALADINO y J. VAES. 2001. Psychological essentialism and the differential attribution of uniquely human emotions to ingroups and outgroups. European Journal of Social Psychology, 31, 395-411.

LEYENS, J. P., P. M. PALADINO, R. RODRIGUEZ-TORRES, J. VAES, S. DEMOULIN y A. RODRIGUEZ-PEREZ. 2000. The emotional side of prejudice: The attribution of secondary emotions to ingroups and outgroups. Personality and Social Psychology Review, 4, 186-197.

MANCHI, M., J. CHEN, G. ROISMAN \& Y. HONG. 2007. Implications for Bicultural Individuals' Cognition and Physiological Reactivity. Psychological science,Vol. 18(4) 341 - 343.

MEDIN, D.L. 1989. Concepts and conceptual structure. American Psychologist, 44, 1469-1481.

MURPHY, G. L. y D. L. MEDIN. 1985. The role of theories in conceptual coherence. Psychological Review. 92, $289-316$.

PRENTICE, D \& D. MILLAR. 2007. Psychological essentialism of human categories. Current directions in psychological science,Vol. 16(4) 202 - 206.

ROTHBART, M. y M. TAYLOR. (1992). Category labels and social reality: Do we view social categories as natural kinds? En K.Semin y A. Fiedler (Eds.), Language, interaction and social cognition (pp. 11-36). London: Sage.

SHERIF, M. 1948. An outline of social psychology. New York: Harper and Row.
TAJFEL, H. 1981. Human groups and social categories. Cambrige: University Press.

TAJFEL, H., M. G. BILLIG, R. P. BUNDY y C. FLAMENT. 1971. Social categorization and intergroup behaviour. European Journal of Social Psychology, 1, 149-178.

VAES, J., M. P. PALADINO y J. LEYENS. P. 2002. The lost e-mail: Prosocial reactions induced by uniquely human emotions. British Journal of Social Psychology, 41, 521-534.

WILLIAMS, M. \& J. EBERHARDT. 2008. Biological Conceptions of Race and the Motivation to Cross Racial Boundaries Journal of Personality and Social Psychology. Vol. 94, No. 6, 1033-1047.

YZERBYT, V. y A. ROGIER. 2001. Blame it on the group: Entitativity, subjective essentialism and social attribution. In J.Jost \& B. Major (Eds.), The psychology of legitimacy: Emerging perspectives on ideology, justice, and intergroup relations (pp. 103-134). New York: Cambridge University Press.

YZERBYT, V., O. CORNEILLE y C. ESTRADA. 2001. The interplay of subjective essentialism and entitativity in the formation of stereotypes. Personality and Social Psychology Review, 5, 141-155.

YZERBYT, V., C. ESTRADA, O. CORNEILLE, E. SERON y S. DEMOULIN. 2003. Subjective essentialism in action: Self anchoringand social control as consequences of fundamental social divides. En V. Yzerbyt, C. M. Judd, y O. Corneille (Eds.), he psychology of group perception: Contributions to the study of homogeneity, entitativity, and essentialism. Psychology Philadelphia press.

YZERBYT, V., S. J. ROCHER y G. SCHADRON. 1997. Stereotypes as explanations: A subjective essentialistic view of group perception. En R.Spears, P. J. Oakes, N. Ellemers, y S. A. Haslam (Eds.), The Social Psychology of Stereotyping and Group Life. Blackwell Oxford published.

YZERBYT, V. y G. SCHADRON. 1996. Connaître et juger autri: Une introduction à la cognition sociale. Presses Universitaires de Grenoble. 